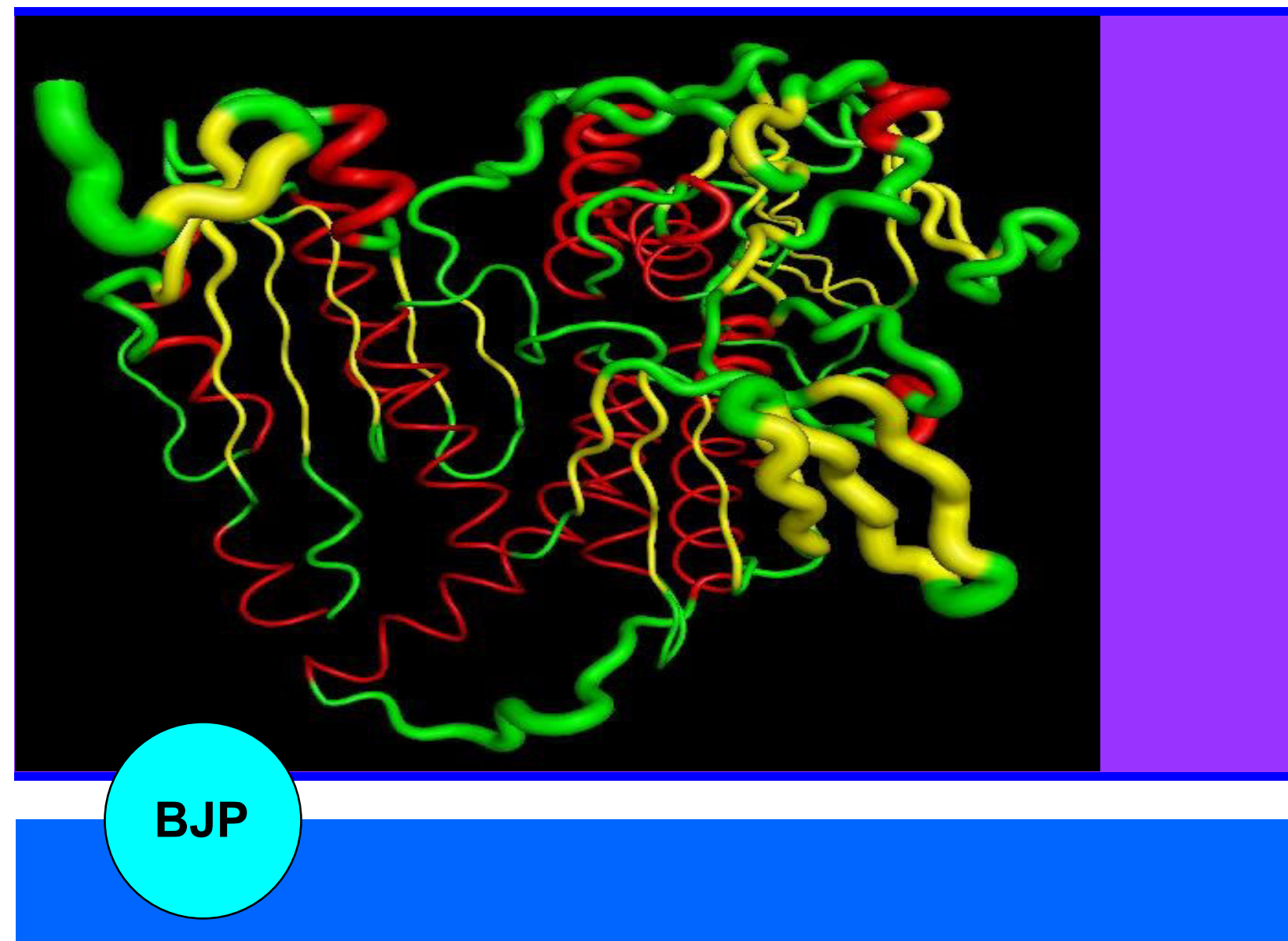

Bangladesh Journal of Pharmacology

Research Article

Molecular docking study on the interaction between trypanothione reductase and mangiferin for antileishmanial activity 


\title{
Molecular docking study on the interaction between trypanothione reductase and mangiferin for antileishmanial activity
}

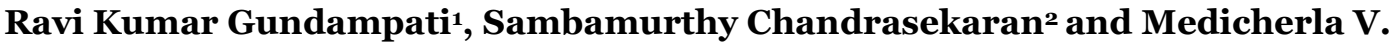 \\ Jagannadham 1
}

\author{
${ }^{1}$ Molecular Biology Unit, Institute of Medical Sciences, Banaras Hindu University, Varanasi 221 005, India; \\ ${ }^{2}$ Department of Animal Sciences, University of Hyderabad, Prof C.R. Rao Road, Gachibowli 500 046, Hyderabad, \\ AP, India.
}

\begin{tabular}{|c|c|}
\hline \multicolumn{2}{|l|}{ Article Info } \\
\hline Received: & 20 December 2012 \\
\hline Accepted: & 22 December 2012 \\
\hline Available Online: & 31 December 2012 \\
\hline \multicolumn{2}{|c|}{ DOI: 10.3329/bjp.v8i1.13034 } \\
\hline \multicolumn{2}{|c|}{$\begin{array}{l}\text { Cite this article: } \\
\text { Gundampati RK, Chandrasekaran S, } \\
\text { Jagannadham MV. Molecular docking } \\
\text { study on the interaction between } \\
\text { trypanothione reductase and man- } \\
\text { giferin for antileishmanial activity. } \\
\text { Bangladesh J Pharmacol. 2013; 8: } 40- \\
43 \text {. }\end{array}$} \\
\hline
\end{tabular}

\begin{abstract}
Mangiferin was found to bind at active site of Leishmania infantum Try $\mathrm{R}$ with lowest binding energy and RMSD values to be $-9.2 \mathrm{Kcal} / \mathrm{Mol}$ and 2.0 respectively. Docking analysis of Try $\mathrm{R}$ with ligand enabled us to identify specific residues viz. Phe-203, Glu-202, Asp-218, Pro-336, Try-221 and Phe270 , within the Try $\mathrm{R}$ binding pocket to play an important role in ligand binding affinity. The availability of Try $\mathrm{R}$ built model, together with insights gained from docking analysis will promote the rational design of potent and selective Try $\mathrm{R}$ inhibitor as antileishmanial therapeutic. The study contributes towards understanding mechanism of antileshmanial effect of the mangiferin. We have surveyed the available literature to summarize the inhibition of Try $\mathrm{R}$ activity of this natural compound. Thus on the basis of our in silico studies we hypothesize that this compound into mangiferin can be inhibitory effect on against leishmaniasis.
\end{abstract}

\section{Introduction}

Trypanosomatids cause various lethal forms of tropical human diseases including Leishmaniasis, which is caused by over twenty different species of Leishmania parasite (e.g., L. donovoni, L. infantum, L. major, L. mexicana, etc). Leishmaniasis causes several clinical disabilities like disseminated visceral infection (Kala-azar). Vector of the disease is female Phlebotomine sandfly, a dipteran, which transmits the parasite to human during blood sucking (Myler and Fasel, 2008). This disease is endemic in low-income population of Central and South American countries (Tempone et al., 2005). Commonly available drugs for Leishmaniasis have severe side effects, high cost and low efficacy (Shukla et al., 2010).

Trypanothione reductase (E.C.1.6.4.8) is a member of the disulfide oxidoreductase family of enzymes (Fairlamb and Cerami, 1992) that presents an attractive target for the development of the new drugs by rational inhibitor design. Tyr R is an NADPH-dependent flavoprotein unique to protozoan parasites from the genera Trypanosoma and Leishmania. Try $\mathrm{R}$ has long constituted an attractive target for chemotherapeutic research in relation to leishmaniasis and other trypanosomatid caused diseases since there are numerous evidences indicating that the enzyme is essential for parasite survival as well as specific in leishmania has not been found in human (Dumas et al., 1997; Tovar et al., 1998).

Mangiferin bioactivities have been related with radical scavenging (Miliauskas et al., 2004) as well as inhibition of oxidative stress (Murunganandan et al., 2005) and complex formation with Fe (III) (Ghosal et al., 1996) where knowledge regarding its fundamental chemical 
behavior could be helpful for a better understanding of its biological pathways. However, there is little information related to quantum mechanics for this molecule (Zhang, 1999) and almost none about docking studies, essential to understand its mechanisms of biological action.

Mangiferin is a plant product of mangifera indica which has been reported to have multiple pharmacological potentials like anti-oxidant activity, immunomodulatory effect, anti inflammatory effect, effect of improving dyslipidemia, therapeutic effect on periodontal disease and antidiabetic effect on both type 1 as well as type 2 diabetes (Dar et al., 2005; Guha et al., 1996; Bhowmik et al., 2009; Niu et al., 2012; Duang et al., 2011).

The detailed in silico analyses of probable inhibition as well as interaction of the models were performed with high binding affinity. However there is no conclusive report as to whether the antileishmanial activity of the mangiferin. In the present study, the structural models of the mangiferin in the trypanothione reductase binding sites has been carried out, which may facilitate further development of more potent antileishmanial agents. Mangiferin might be a promising additive in combined drug inhibitor of trypanothione reductase. Protein-lingand docking was performed between the molecular models of L. infantum Try R and Mangiferin. The studies presented in this medical hypothesis will be useful to design molecules that may have antileishmanial activity.

\section{Materials and Methods}

Python 2.7-language was downloaded from www.python.com, Cygwin c: \program and Python 2.5 were simultaneously downloaded from www.cygwin.com, Molecular graphics laboratory (MGL) tools and AutoDock 4.2 was downloaded from www.scripps.edu, Discovery studio visualizer 2.5.5 was downloaded from www.accelerys.com.

The docking of Mangiferin into the binding site of the Try R protein was explored using AutoDock software, which has been shown to be powerful tools for molecular recognition. To validate the molecular modeling programs, we first evaluated the docking accuracies of AutoDock by docking with known Try $\mathrm{R}$ inhibitor, mangiferin into the binding site.

\section{Protein preparation for docking}

The 3D structure of Leishmania Infantum Try R (PDB ID: 2jk6) was downloaded from Protein Data Bank (PDB) (http://www.pdb.org/pdb/home/home.do), before initiating the docking simulations, all non-protein molecules were removed from Try $\mathrm{R}$ (Figure 1); for any alternative atom locations only the first location was retained. All the docking calculations were performed by using AutoDock 4.0. Try $\mathrm{R}$ was modified by adding polar hydrogen's and then kept rigid in the docking process, whereas all the torsional bonds of ligands were set free by Ligand module in AutoDock tools. Various inhibitors provide an excellent basic for using structurebased approaches for the discovery of new inhibitors.

\section{Ligand preparation for docking}

The flavonoid ligand like Mangiferin was built using Chemsketch and optimized using "Prepare Ligands" in the AutoDock 4.2 for docking studies. The optimized ligand molecules were docked into refined Trypanothione reductase model using "LigandFit" in the AutoDock 4.2

Binding sites of these complexes were identified as described previously. The conformation of binding site was constructed manually to accommodate with mangiferin. The validation of the docking accuracy was done by docking of the Mangiferin into its binding site of Trypanothione reductase. AutoDock binding affinities of the mangiferin into Trypanothione reductase were evaluated by the binding free energies $(\mathrm{kcal} / \mathrm{mol})$, inhibition constants (Ki), hydrogen bonds, and RMSD values.

\section{Molecular docking}

AutoDock 4.2 suite was used as molecular-docking tool in order to carry out the docking simulations. The Auto Dock 4.2 program was used to investigate ligand binding to structurally refined Try $\mathrm{R}$ model using a default grid spacing of $0.375 \AA$ and the grid points in $X$, $\mathrm{Y}$ and $\mathrm{Z}$ axis were set to $60 \times 60 \times 60 \AA$. The search was based on the Lamarckian genetic algorithm (Oprea et al., 2001) and the results were analyzed using binding energy. For each ligand, a docking experiment consisting of 100 stimulations was performed and the analysis was based on binding free energies and root mean square deviation (RMSD) values, and the ligand molecules were then ranked in the order of increasing docking energies.

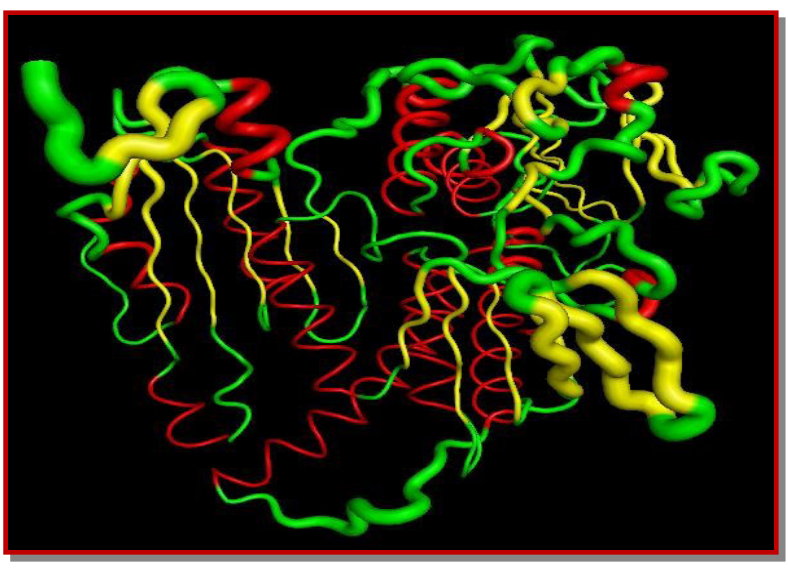

Figure 1: The modelled structure of trypanothione reductase receptor 


\section{Results and Discussion}

The docking of mangiferin into the binding site of the Try-R protein was explored using AutoDock software, which has been shown to be powerful tools for molecular recognition. To validate the molecular modeling programs, we first evaluated the docking accuracies of AutoDock by docking with known Try $\mathrm{R}$ inhibitor, mangiferin into the binding site. The 3D structure of Leishmania Infantum Try R (PDB ID: 2jk6) was downloaded from Protein Data Bank (PDB) (http:// www.pdb.org/pdb/home/home.do), in these mangiferin as ligand for our in silico studies shown in Figure 2.

Molecular docking methods are commonly used for predicting binding modes to proteins and energies of ligands (Bikadi and Hazai, 2009). Docking was accomplished employing AutoDock 4.0 which is a suite of automated docking tools was used to predict the affinity, activity, binding orientation of mangiferin to our target protein molecule Try R. Analysis was based on Etotal or free energy of binding, lowest docked energy, and calculated RMSD values. For each approach, the number of hits, the RMSD value of the best hit (with the lowest RMSD) based on shape complementarity are listed in Table I. Mangiferin was found to bind at active site of L. infantum Try $\mathrm{R}$ with lowest binding energy and RMSD values to be -9.2 Kcal/Mol and 2.0 respectively (Table I). Free energy of binding is calculated as a sum of four energy terms of intermolecular energy (vanderwaal, hydrogen bond, desolvation energy and electrostatic energy), total internal energy, torsional free energy and unbound system energy. The major interactions shown in the Try $\mathrm{R}$ binding site are the important $\mathrm{H}$-bonds with Phe-203 (H Bond 2.07 ̊̊), Glu-202(H Bond $2.12 \AA$ ), Asp218(H Bond $1.97 \AA$ ), Pro-336(H Bond $1.83 \AA$ ), Try-221(H Bond $2.04 \AA$ ), and Phe-270 (H Bond $2.75 \AA$ ). The docking of L. infantum Try $\mathrm{R}$ and mangiferin is shown in Figure 3. Our in silico experiments demonstrate that mangiferin binds Try $R$, and also is itself inhibits its

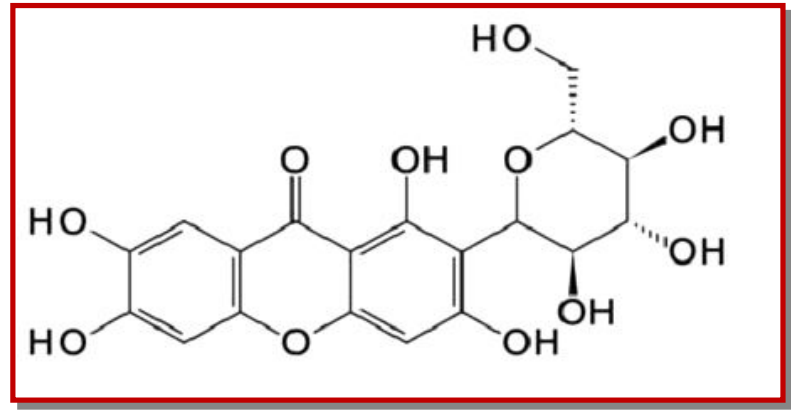

Figure 2: Parental structure of mangiferin

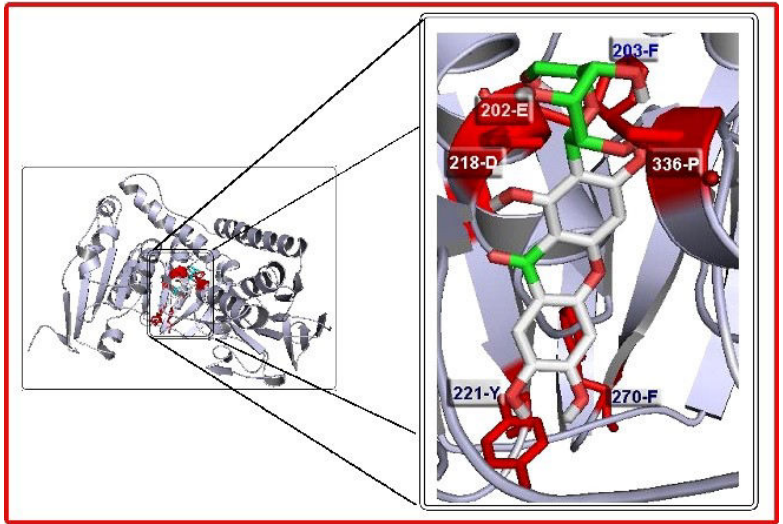

Figure 3: In silico analysis of the interaction of trypanothione reductase with mangiferin. Try R amino acids Phe-203, Glu202, Asp-218, Pro-336, Try-221 and Phe270 from hydrogen bonds with mangiferin

function and thus may act as a drug.

A detailed literature survey yielded that the inhibitory pathway has to be considered as a potential drug target against the parasitic protozoan species of Trypanosoma and Leishmania infantum Try $\mathrm{R}$ using bioinformatics tools. We can hypothesize that mangiferin may be considered to play an important role in inhibiting the Try $\mathrm{R}$ activity and progression of leishmaniasis. This mangiferin can be promising candidate for the development towards the design of anti-leishmanial drug as therapeutic compound.

\section{Table I}

\section{Docking results of mangiferin molecules docked on to trypanothione reductase model}

\begin{tabular}{|c|c|c|c|c|c|}
\hline $\begin{array}{l}\text { SL. } \\
\text { No }\end{array}$ & Lead molecule & Run & RMSD & $\begin{array}{l}\text { Estimated free energy } \\
\text { of binding }(\mathrm{kcal} / \mathrm{mol})\end{array}$ & $\begin{array}{l}\text { Estimated inhibition constant, Ki } \\
n \mathrm{nM} \text { or } \mu \mathrm{M} \quad[\text { Temp }=298.2 \mathrm{~K}]\end{array}$ \\
\hline 1 & Mangiferin-parental compound & 40 & 1.2 & -9.2 & $191.4 \mathrm{nM}$ \\
\hline 2 & & 9 & 1.6 & -7.4 & $3.7 \mu \mathrm{M}$ \\
\hline 3 & & 6 & 2.0 & -9.2 & $198.6 \mathrm{nM}$ \\
\hline 4 & & 13 & 1.9 & -7.1 & $6.1 \mu \mathrm{M}$ \\
\hline 5 & & 5 & 1.6 & -6.7 & $13.2 \mu \mathrm{M}$ \\
\hline 6 & & 16 & 1.9 & -6.6 & $14.2 \mu \mathrm{M}$ \\
\hline 7 & & 29 & 1.1 & -6.3 & $24.2 \mu \mathrm{M}$ \\
\hline 8 & & 39 & 1.7 & -4.8 & $300.4 \mu \mathrm{M}$ \\
\hline
\end{tabular}




\section{Conclusion}

The results of the present study clearly demonstrated the in silico molecular docking studies of mangiferin with Try $\mathrm{R}$ enzyme exhibited binding interactions and warrants further studies needed for the development of potent trypanothione reductase inhibitor for the treatment of leishmaniasis.

\section{Acknowledgements}

RKG wants to thank D. S. Kothari Post Doctoral Fellowship, University Grants Commission, Government of India for financial support and the Molecular Biology Unit, Institute of Medical Sciences, Banaras Hindu University, Varanasi, India for providing laboratory and technical support, financial assistance.

\section{References}

Bhowmik A, Ali Khan L, Akhter M, Rokeya B. Studies on the antidiabetic effects of Mangifera indica stem-barks and leaves on nondiabetic, type 1 and type 2 diabetic model rats. Bangladesh J Pharmacol. 2009; 4: 110-14.

Bikadi Z, Hazai E. Application of the PM6 semi-empirical method to modeling proteins enhances docking accuracy of AutoDock. J Cheminform. 2009; 11: 1-15.

Dar A, Faizi S, Naqvi S, Roome T, Zikr-ur-Rehman S, Ali M, Firdous S, Moin ST. Analgesic and anti-oxidant activity of mangiferin and its derivatives: The structure activity relationship. Biol Pharm Bull. 2005; 28: 596-600.

Duang X, Wang Q, Zhou X, Huang D. Mangiferin: A possible strategy for periodontal disease to therapy. Medical Hypotheses. 2011; 76: 486-88.

Dumas C, Ouellette M, Tovar J, Cunningham ML, Fairlamb AH, Tamar S, Olivier M, Papadopoulouet B. Disruption of the trypanothione reductase gene of Leishmania decrease its ability to survive oxidative stress in macrophages. EMBO J. 1997; 16: 2590-98

Fairlamb AH, Cerami A. Metabolism and function of trypano- thione in the kinetoplastida. Ann Rev Microbiol. 1992; 46: 695-729.

Ghosal S, Rao G, Saravanan V, Misra S, Rana D. A plausible chemical mechanism of the bioactivities of mangiferin. Indian J Chem B Org. 1996; 35B: 561-66.

Guha S, Ghosal S, Chattopadhyay U. Antitumor, immunemodulatory and anti-HIV effect of mangiferin, a naturally occurring glucosylxanthone. Chemotherapy 1996; 42: 443-51.

Miliauskas G, Venskutonis PR, Van Beek TA. Screening of radical scavenging activity of some medicinal and aromatic plant extracts. Food Chem. 2004; 58: 231-37.

Murunganandan S, Lal J, Gupta PK. Immunotherapeutic effects of mangiferin mediated by the inhibition of oxidative stress to activated lymphocytes, neutrophils and macrophages. Toxicology 2005; 215: 57-68.

Myler PJ, Fasel N. Leishmania: After the genome. Norfolk, UK, Caister Academic Press, 2008.

Niu Y, Li S, Na L, Feng R, Liu L, Ying L, Changho S. Mangiferin decreases plasma free fatty acids through promoting its catabolism in liver by activation of AMPK. PLoS ONE. 2012; 7: 1-8.

Oprea TI, Davis AM, Teague SJ, Leeson PD. Is there a difference between leads and drugs? A historical perspective. J Chem Inf Comput Sci. 2001; 41: 308-15.

Shukla AK, Singh BK, Patra S, Dubey VK. Rational approaches for drug designing against leishmaniasis. Appl Biochem Biotechnol. 2010; 160: 2208-18.

Tempone AG, Borboremab SE, de Andrade HF, Amorim Gualda NC, Yogi A, Salerno Carvalho C, Bachiega D, Lupo FN, Bonotto SV, Fischer DCH. Antiprotozoal activity of Brazilian plant extracts from isoquinoline alkaloid-producing families. Phytomedicine 2005; 12: 382-90.

Tovar J, Cunningham ML, Smith AC, Croft SL, Fairlamb AH. Down-regulation of Leishmania donovani trypanothione reductase by heterologous expression of a trans-dominant mutant homologue: Effect on parasite intracellular survival. Proc Natl Acad Sci. 1998; 95: 5311-16.

Zhang HY. AM1 calculation study of scavenging effect of Chinonin on free radicals. Acta Pharmacol Sin. 1999; 20: 55558 .

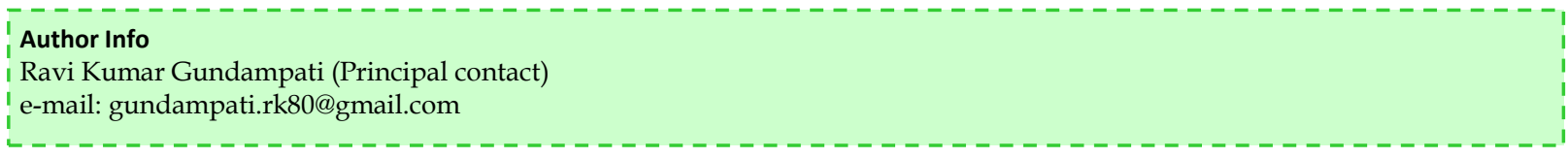

